\title{
Neuropsicologia Clínica: Notas Históricas, Fundamentos Teórico-Metodológicos e Diretrizes para Formação Profissional
}

\author{
José Roberto Wajman* (1) \\ Universidade Federal de São Paulo, Escola Paulista de Medicina (UNIFESP-EPM), São Paulo, SP, Brasil
}

\begin{abstract}
RESUMO - A Neuropsicologia se concentra em disciplinas oriundas da interface entre a neurociência e a psicologia cognitiva, e sua pesquisa converge da relação entre o cérebro e o comportamento. Como se tem observado em diversos centros de referência no Brasil, a difusão da Neuropsicologia vem ocorrendo de maneira vertiginosa, embora o estabelecimento de diretrizes de educação e treinamento ainda se encontrem despadronizados. O presente artigo descreve o desenvolvimento histórico da Neuropsicologia, enfocando seus fundamentos teóricos e metodológicos, sua condição regulatória atual, além de perspectivas futuras na criação de diretrizes para capacitação profissional. Pesquisas vêm demonstrando uma tendência em caracterizar a atividade do neuropsicólogo nos mais diversos locais nos quais a neuropsicologia é ensinada, praticada e difundida. No Brasil, faz-se necessária uma taxonomia acerca do ofício do profissional especialista em neuropsicologia, seja em relação à sua formação ou sua atuação profissional.
\end{abstract}

PALAVRAS-CHAVE: neuropsicologia, história da psicologia, formação profissional

\section{Clinical Neuropsychology: Historical Notes, Theoretical-Methodological Foundations and Guidelines for Professional Training}

\begin{abstract}
Neuropsychology focuses on disciplines arising from the interface between neuroscience and cognitive psychology, and its research converges on the relationship between the brain and behavior. As has been observed in several reference centers in Brazil, the spread of Neuropsychology has been occurring in a dizzying way, although the establishment of education and training guidelines are still out of standard. This article describes the historical development of Neuropsychology, focusing on its theoretical and methodological foundations, its current regulatory condition, as well as future perspectives in the creation of guidelines for professional training. Studies have shown a tendency to characterize the activity of the neuropsychologist in the most diverse fields in which it is taught, practiced, and diffused. In Brazil, it is necessary a taxonomy about the profession of the specialist in neuropsychology, whether in relation to his training or his professional performance.
\end{abstract}

KEYWORDS: neuropsychology, history of psychology, professional education

A Neuropsicologia, como a compreendemos atualmente, diz respeito à disciplina de base teórica e aplicação prática - clínica e experimental -, que resulta da interface entre neurociência e psicologia cognitiva, dispondo em seu cerne o estudo das relações entre cérebro e comportamento (Gounden et al., 2017). Adicionalmente, a Neuropsicologia descreve as etapas de aquisição e desenvolvimento das habilidades neurocognitivas durante as fases críticas de maturação sendo que, por vezes, esses modelos explicativos para tais associações decorrem das variações provocadas por lesões e/ou patologias, de forma inata ou adquirida e de curso reversível, sequelar e/ou progressivo.

A Neuropsicologia, como área de conhecimento, remonta a época dos antigos egípcios (e, mais adiante, de gregos e romanos). A mesma, tem seu nome originalmente cunhado por Sir William Osler, no século XX, durante uma

*E-mail: betowajman@usa.com

- Submetido: 03/11/2017; Revisado: 04/07/2018; Aceito: 30/07/2018. 
conferência na escola de medicina do renomado hospital Johns Hopkins. O termo surgiu novamente na obra The Organization of Behavior: A Neuropsychological Theory, do Canadense Donald Olding Hebb, evidenciando sua aplicação no contexto de uma psicologia fisiológica que fosse capaz de responder questões sobre os fundamentos neurais do comportamento humano (Mäder, 1996).

Em busca de origens e traçando uma rota histórica, observa-se na literatura que papiros adquiridos por Edwin Smith em Luxor, datados dos séculos XVI e XVII a.C., já documentavam o conhecimento destes povos sobre as funções cerebrais. Nos séculos II e III a.C., Galeno, Herófilo e Hipócrates rabiscavam teorias sobre a relação da anatomia cerebral com afetos, mente e processos cognitivos, como por exemplo, a linguagem. À época, diferentes pensadores admitiam o cérebro como órgão do pensamento e das sensações, os ventrículos como sítios de onde emergiam os processos cognitivos e a massa cerebral responsável pelas atividades da mente (Bruce, 1985).

Transcorrida a estéril era da Idade Média e já nos séculos XVII e XVIII, pensadores decidiram-se dedicar-se aos estudos anatomofuncionais do cérebro. Após famosa menção de René Descartes, em meados dos anos 1700, David Hartley (pai da psicologia associacionista) propõe através de seus experimentos que nervos, medula espinhal e cérebro constituem uma mesma estrutura essencial. A seguir, na década de 1800, em paralelo às hipóteses de Charles Bell e Gustav Theodor Fechner sobre a diferenciação entre vias motoras e sensitivas, Jean Pierre Flourens e Marc Bouillard descrevem diferentes centros cerebrais para diferentes domínios cognitivos, sendo seu funcionamento em rede (Boring, 1950).

Uma profícua discussão sobre a localização cerebral foi inaugurada por Franz Joseph Gall, Paul Broca e Carl Wernicke pela observação anatômica de protuberâncias cranianas e sua relação com traços de personalidade, distúrbios específicos da fala e dominância inter-hemisférica, além da conjectura sobre vias de associação entre diferentes regiões cerebrais. Na França e em Londres, simultaneamente, primeiros passos eram ensaiados por Alfred Binet e Francis Galton na busca de métodos de avaliação da inteligência (Spreen \& Strauss, 1991).

Na história contemporânea, têm-se como vanguardistas dos estudos em Neuropsicologia expoentes como Karl Spencer Lashley, responsável pela Teoria da Equipotencialidade, para a qual as alterações do comportamento dependiam mais da quantidade de massa do que de sua localização, Paul MacLean, pai da Teoria do Cérebro Trino, que versa sobre o cérebro dividido em: reptiliano, mamífero inferior e racional, além de Hans Lukas Teuber, Lawrence Weiskrantz e Tim Shallice, responsáveis pelo princípio da dissociação entre funções cognitivas (Carrilho, 2009).

Adicionalmente, temos: Arthur Lester Benton, pesquisador de síndromes afásicas e criador de diversos testes neuropsicológicos; Brenda Milner, dedicada ao estudo de pacientes epilépticos, submetidos à cirurgia dos lobos temporais; Ronald Myers e Roger Sperry, estudando pacientes e animais submetidos à calosotomia; Norman Geschwind, proponente do termo síndrome desconectiva; e Theodor Meynert, desbravando as fibras de associação hemisféricas e inter-hemisféricas, além de vários outros renomados colaboradores (Carrilho, 2009).

Em vista de um crescente interesse no estudo de quadros afásicos, neurologia e psicologia soviéticas não apenas contribuíram para descrever seus sintomas centrais, mas também forneceram significativas contribuições para explicar as bases fisiológicas dos distúrbios de linguagem. Mais adiante e apesar de ainda influenciado pela herança fisiológica de Sechenov e Ivan P. Pavlov, Lev Vygotsky procurou substituir as doutrinas localizacionista e globalista (Vygotsky, 1991). Vygotsky, desta maneira, propôs três instâncias principais para aquilo que entendia como "Funções Corticais Superiores": (a) associação entre sistemas funcionais cambiantes - plasticidade; (b) funções dinâmicas resultantes da convergência entre funções básicas; e (c) entendimento e percepção real de nosso psiquismo.

Os estudos de Alexander Romanovich Luria, embasados no trabalho de Vygotsky com pacientes neurológicos russos a partir da década de 1920, representam um marco histórico para a Neuropsicologia. Luria preconizava uma disciplina que oferecesse, simultaneamente, paralelismo entre aspectos fisiológicos e neurológicos, sem que deles emergisse uma relação unicamente causal e, além disso, que jamais abrisse mão de um entendimento "humanizado" das mais diversas apresentações clínicas investigadas. Não obstante, Luria contribui igualmente no que toca o avanço técnico-metodológico realizado via exame clínico: manejos outrora considerados elementares, porém agora calcados no tríplice conceito de "Funções Corticais Superiores", passam a conduzir a práxis observacional da investida neuropsicológica (Luria, 2005).

Luria (2005) se destacaria por sua teoria das três unidades básicas, ou sistemas funcionais, na qual constariam: (I) Uma unidade atencional, ou de regulação do tônus e vigília que envolve camadas do córtex e o sistema reticular ativador; (II) Uma unidade de codificação para obter, processar e armazenar as informações que chegam do mundo exterior (e dos aparelhos de seu próprio corpo) localizada nos lobos occipital, temporal e parietal e (III) Uma unidade de planificação para programar, regular e verificar a atividade mental. Esse terceiro bloco (ou unidade), localizado basicamente nas porções dorsolaterais e ventromediais dos lobos frontais, seria quem elabora programas de comportamento, assegura e regula sua realização e participa do controle de seu cumprimento.

Conforme definida à luz de Luria (2005), a Neuropsicologia representa uma ciência dedicada a estudar a expressão comportamental das disfunções cerebrais e sua práxis está calcada em uma série de princípios fundamentais acerca da cognição. A mesma pode, portanto, 
ser segmentada em ao menos quatro grandes módulos: (1) funções receptivas: que envolvem a capacidade de perceber, selecionar, classificar e integrar informações; (2) memória e aprendizagem: que se referem ao armazenamento e recuperação de dados; (3) processamento do pensamento: que diz respeito à permanente organização e reorganização das informações dentro de um sistema de alimentação e retroalimentação e (4) funções expressivas: meios pelos quais as informações podem ser acessadas, recuperadas e comunicadas. (Lezak, 1995).
Desse modo, o presente artigo (em formato de estudo teórico realizado por meio de revisão não sistemática da literatura) possui como objetivo principal apresentar os aspectos fundamentais da Neuropsicologia clínica, partindo desde seu desenvolvimento conceitual até modelos taxonômicos para caracterização da prática profissional, passando por notas históricas de expressão, informes sobre legislação e regulamentação normativa de classe, desafios diante do panorama sociocultural e tecnológico, chegando, finalmente, em uma proposta de diretriz unificada, em prol do avanço social e científico da Neuropsicologia brasileira.

\section{PIONEIROS DA NEUROPSICOLOGIA: RETRATO BRASILEIRO E SUA ORGANIZAÇÃO COMO ESPECIALIDADE}

Com publicação há menos de duas décadas no proeminente Journal of the International Neuropsychological Society, pela primeira vez foi apresentado um rascunho de tratado a respeito da organização profissional e de aspectos históricos acerca da Neuropsicologia e de suas implicações práticas (Rourke \& Murji, 2000). Nesse artigo, encontram-se informações valiosas sobre a primeira reunião científica da International Neuropsychological Society (INS), em 1967, conjuntamente ao encontro anual da American Psychological Association (APA), ocorrido em Washington, EUA.

A finalidade desta junta, à ocasião, seria a pesquisa abrangente dos achados clínicos secundários a processos neuropatológicos e suas manifestações sobre o comportamento humano. O conteúdo da obra resulta em um relatório detalhado dos feitos e atuações desta assembleia logo à ocasião de sua fundação, oferecendo um apanhado cronológico dos ocorridos entre o período de 1965 a 1985 pudesse ser examinada futuramente. $O$ expediente inaugural aponta para o início das articulações para a criação de uma sociedade que congregasse profissionais e temas afins. A última, data e comemora a afiliação da sociedade ao Journal of Clinical and Experimental Neuropsychology (Kapczinski \& Narvaez, 2008).

Em nosso meio, um dos precursores na aplicação dos conceitos neuropsicológicos foi o médico Antonio Frederico Branco Lefèvre (1916-1981), reconhecido como um dos pais da neurologia infantil nacional. Preocupado com o fato que a neurologia, isoladamente, não seria suficiente para uma especialidade que lidava com o desenvolvimento do sistema nervoso, Lefèvre julgou importante para sua formação conhecer e estudar também problemas de ordem psicológica (Lefèvre, 1985). À mesma época, Beatriz Helena Withaker Ferreira, nascida na cidade de São Carlos, iniciava seus estudos em Filosofia e Psicologia. Após um retiro na Europa, especializou-se em Psicologia Clínica no Institut de Psychologie de l'Université de Paris. Em seu regresso ao Brasil, iniciou suas atividades na Clínica Neurológica do Hospital das Clínicas da Faculdade de Medicina da USP, onde conheceu o Prof. Antonio Lefèvre que, a seguir, se tornaria seu marido. Juntamente com o Professor Lefèvre, Beatriz Branco Lefèvre estreava nossa Neuropsicologia, ao final da década de 1960 (Dias, 2013).

Já no final da década de 1980, por aguerrida atuação e empenho de Norberto Rodrigues e Jayme Antunes Maciel Jr., se dá a inauguração formal da Sociedade Brasileira de Neuropsicologia (SBNp), em Assembleia Geral da Academia Brasileira de Neurologia, durante o XIII Congresso Brasileiro de Neurologia, em São Paulo. Nessa época, foi determinado que a alcunha "Sociedade Brasileira de Neuropsicologia" adequadamente caracterizava os princípios desta congregação na arregimentação multi e interdisciplinar dos colegas que atuavam com ensino e pesquisa em psicologia cognitiva. Para lavrar a decisão, a ata da fundação da SBNp foi registrada no $4^{\circ}$ Cartório de Registro de Títulos e Documentos - Cartório Medeiros, em São Paulo, como uma sociedade civil, sem fins lucrativos.

Já no final dos anos 1980, formou-se em São Paulo uma comissão multidisciplinar com profissionais dos mais diversos grupos e instituições que trabalhavam na área da Neuropsicologia, para juntos organizarem o I Congresso Brasileiro de Neuropsicologia e, também, o II Congresso Latino-americano de Neuropsicologia. Nesse evento, ocorrido em 1991, compareceram ilustres colegas de outros países da América Latina e da Europa. A partir desse marco histórico-científico, instalou-se a tradição de ser lançado, após cada congresso da SBNp, um livro contendo as palestras proferidas. Como resultado, foram editados dois livros: Temas em Neuropsicologia (vol. I) e Temas em Neurolinguística (vol. II). A área de atuação da Neuropsicologia no Brasil, assumida pela SBNp, foi registrada por Norberto Rodrigues no capítulo intitulado: "Neuropsicologia: Uma Disciplina Científica" (Mueller, 1968).

Assertiva e declaradamente, a SBNp compreende que a Neuropsicologia é uma ciência multi, inter e transdisciplinar. De acordo com a própria associação, seu escopo visa privilegiar parcerias, além da troca de conhecimentos e de experiências entre as diversas áreas da 
saúde e da educação, respeitando seus limites de atuação, de acordo com a especialização exigida em cada uma dessas profissões. Portanto, no ano de 2007, de acordo com o novo Código Civil, a alcunha de SBNp foi convertida em Associação Brasileira Multidisciplinar de Neuropsicologia. Já no primeiro semestre de 2008, a SBNp organizou o Simpósio "Avaliação em Neuropsicologia: estudos atuais na normatização e validação de testes no Brasil", com foco na discussão sobre as diferentes ferramentas de Avaliação em Psicologia e, a partir dai, vem consecutivamente agregando filiados (dentre eles, estudantes) e organizando eventos entre acadêmicos, psicólogos formados e demais pesquisadores da área.

Atualmente a sociedade possui diversos associados ativos, contando com o apoio de mais de vinte instituições universitárias públicas e privadas distribuídas nas regiões sul, sudeste e nordeste do país. Seu corpo de membros é constituído por psicólogos, fonoaudiólogos, terapeutasocupacionais, médicos, pedagogos, psicopedagogos, musicoterapeutas, linguistas, biólogos, professores universitários e estudantes. Sua diretoria é democraticamente eleita para mandatos bienais e seu portfólio é constituído de
15 livros publicados na área, sendo que boa parte resulta do agregado de produções provenientes de eventos científicos realizados local e regionalmente.

A exemplo da SBNp, com endereço na cidade de São Paulo, o Instituto Brasileiro de Neuropsicologia e Comportamento (IBNeC), fundado em 2009 e situado no Rio de Janeiro, é uma associação científica que congrega pesquisadores, profissionais e estudantes de psicologia e áreas afins de todas as regiões do país, com interesse nas diferentes áreas da neurociência e suas intersecções com a psicologia. Adicionalmente, o IBNeC também é um órgão que representa os neuropsicólogos junto às agências de fomento à pesquisa e entidades responsáveis pela avaliação de produtividade científica no país, mantendo, ainda, o propósito de ocupar espaços nos conselhos que regulamentam a profissão da psicologia no Brasil (IBNEC, 2018). No ano de 2013, ocorreu aquele que é considerado até hoje o maior evento científico em Neuropsicologia do país. Reunidas, SBNp e IBNeC organizaram seus tradicionais encontros, a Reunião Anual do $\mathrm{IBNeC}$ e o Congresso Brasileiro de Neuropsicologia da SBNp em um único evento, na cidade de São Paulo.

\section{REGULAMENTAÇÃO PROFISSIONAL, MULTIDISCIPLINARIDADE E AS DIFERENÇAS ENTRE TÍTULO E CERTIFICADO EM NEUROPSICOLOGIA}

De acordo com o Conselho Federal de Psicologia (CFP, 2007), o título de especialista em neuropsicologia é concedido exclusivamente ao psicólogo que possui experiência profissional constatada e declarada das seguintes formas: (a) por meio do tempo de atuação profissional (dois anos), (b) mediante verificação de experiência; curso de especialização reconhecido e/ou (c) prova-concurso para concessão de título. Adicionalmente, todos eles devem estar devidamente credenciados pelo conselho de classe e/ou Ministério da Educação e Cultura. A resolução do CFP, de número 013/2007, outorga o título de especialista em Neuropsicologia ao profissional da psicologia com reconhecida competência junto aos processos intrínsecos à prática da especialidade: avaliar, acompanhar, tratar e desenvolver pesquisa com enfoque na relação entre aspectos psíquicos e fundamentos neurobiológicos, primando por uma ciência baseada em evidências.

Reconhecida como uma das etapas mais importantes do processo neuropsicológico, a avaliação neuropsicológica é - da mesma maneira - restrita ao neuropsicólogo e deve fornecer resultados (parciais e/ou globais, preliminares e/ ou definitivos) em forma de parecer, relatório ou laudo do avaliando. Não obstante, tal documento tem sua importância não somente na descrição quanti-qualitativa dos achados psicométricos, mas também sugestões de acompanhamento e seguimento prospectivo-longitudinal.

Em vista das próprias origens da Neuropsicologia remontarem a fundamentos de uma ciência abrangente acerca do funcionamento do cérebro e modulação do comportamento, anseia-se, como formatação profissional ideal, uma Neuropsicologia multidisciplinar e integrada com as mais diversas áreas de atuação que igualmente compõem as neurociências: Neurologia, Psiquiatria, Fonoaudiologia, Pedagogia, Fisioterapia, Assistência Social, Terapia Ocupacional, Educação Física e Nutrição, dentre outras.

Há tempos esse tema parece suscitar diversas dúvidas no que toca o papel do profissional da área da saúde e da educação que atua com temas em Neuropsicologia: àqueles profissionais com outros cursos de graduação que não o de psicologia, não lhes é deferido o título de neuropsicólogo. Em situações em que esses profissionais (não psicólogos) concluíram e diplomaram-se em cursos de especialização em Neuropsicologia, devidamente reconhecidos pelo CFP, sua certificação se dá no sentido em que o mesmo esteja apto a atuar em parceria com profissionais e/ou instituições certificadas, uma vez acompanhado e/ou supervisionado por um neuropsicólogo assentido e devidamente registrado como tal junto ao seu conselho local. Esses profissionais não psicólogos, por sua vez, recebem o título, outorgado pelo MEC, de reabilitadores cognitivos, e sua atuação é restrita à reabilitação.

Subproduto do CFP, o Sistema de Avaliação de Testes Psicológicos (SATEPSI; CFP, 2018) é um sistema desenvolvido pelo próprio CFP para divulgar informações sobre os testes psicológicos aos profissionais da área e à comunidade. Pode-se obter informações sobre os 
testes psicológicos com parecer favorável e desfavorável verificando a possibilidade de uso do teste na avaliação psicológica/neuropsicológica, bem como da identificação de instrumentos privativos e não exclusivos do psicólogo. Em conclusão, conforme preconizado, o acesso e uso dos testes neuropsicológicos deverá ocorrer de maneira exclusiva e de acordo com a resolução disposta no artigo 13 da lei $\mathrm{N}^{\circ} 4.119 / 62$, que restringe ao psicólogo o uso de técnicas e métodos psicológicos, considerando contravenção penal o exercício ilegal da profissão por profissionais não enquadrados nesta resolução.

De acordo com o SATEPSI (CFP, 2018), a figura do profissional de psicologia deve corresponder a de um especialista com visão sobre a magnitude das ciências biológicas, humanas e sociais; que desempenhe um trabalho técnico e de notória qualidade; calcado sobretudo em fundamentos éticos; voltado para a atuação em equipe, e apto a utilizar da sua formação para a produção e disseminação de seu entendimento nos mais variados contextos que careçam de sua participação em situações que demandam incentivo psicossocial á dignidade humana e á coletividade, como um todo. Desta maneira, á tal profissional é exigido o atendimento e respeito á Resolução CFP n ${ }^{\circ} 002 / 2003$, a fim de completa percepção de que instrumentos de avaliação psicológica poderão tão somente ser empregados em nosso meio uma vez que obtenham parecer favorável do CFP, sem desconsiderar a utilização de medidas desfavoráveis, em condição de pesquisa.

Assim, O CFP compreende existir ao menos um par de modalidades de cursos sobre Avaliação Psicológica: aqueles que introduzem profissionais no ambiente da pesquisa científica e aqueles que desenvolvem as ferramentas psicológicas propriamente ditas. No que tange a difusão de testes outrora julgados desfavoráveis, o CFP recomenda a suspensão do curso em prol da manutenção da qualidade técnica-científica profissional. Para os centros que visam a pesquisa, uma vez adequadamente orientados, os profissionais podem utilizar essas ferramentas valendo-se do princípio de que a investigação científica se faz inexorável ao desenvolvimento profissional da psicologia.

Sobre o perfil do psicólogo brasileiro no tocante à especialidade de Neuropsicologia e seu contato com instrumentos de avaliação, o repartimento, mediante assessoria responsável, aponta não haver dados atualizados sobre a quantidade de psicólogos que trabalham em cada área, ou nos setores público e privado. Entretanto, de acordo com pesquisa IBOPE/MQI realizada no ano de 2004 (IBOPE Opinião, 2004), por meio de 2000 entrevistas telefônicas, do total de 994 psicólogos devidamente inscritos no CFP, 71\% referiram conhecer o sistema de avaliação de testes psicológicos, não havendo diferenças estatisticamente significantes quanto a suas características sociodemográficas e/ou tempo desde a formação/graduação.
Quando questionados sobre a importância das principais ações praticadas por intermédio das comissões do CFP, aproximadamente $38 \%$ apontaram o sistema de avaliação de testes psicológicos como uma iniciativa relevante ao exercício de sua profissão. Como dado adicional, do montante total, metade realizou alguma especialização, ao passo que menos de $10 \%$ ingressou em programas de mestrado ou doutorado.

Em resumo, os métodos neuropsicológicos compreendem não somente testes, mas também procedimentos que têm, como produto, a análise do comportamento e, mais especificamente, das funções cognitivas e suas correlações com as funções e áreas cerebrais. De acordo com os descritores de saúde da Biblioteca Virtual em Saúde da área da Psicologia (Centro Latino-Americano e do Caribe de Informação em Ciências da Saúde, n.d.), testes neuropsicológicos são "testes projetados para a avaliação da função neurológica associada a certos comportamentos. São utilizados no diagnóstico de disfunção ou dano cerebral e dos transtornos ou lesões do sistema nervoso central". Seguindo tal premissa, pode-se dizer que o sucesso da Neuropsicologia no Brasil está diretamente relacionado com a capacidade, dedicação e eficiência dos profissionais da área que, apesar de regulamentada há poucos anos, vem se desenvolvendo e sedimentando terreno cada vez mais fértil (Miotto, 2007).

Em 2017, constavam no diretório eletrônico do CFP 14 centros/cursos/núcleos para formação e certificação do especialista em Neuropsicologia. Destes, sete estão na capital ou no interior de São Paulo (SP), dois no Rio Grande do Sul (RS), dois em Pernambuco (PE), um em Brasília (DF) e outro no estado do Rio de Janeiro (RJ) (http://site.cfp.org. $\mathrm{br} /$ servicos/titulo-de-especialista/cursos-credenciados/) ${ }^{1}$. Há também os concursos de provas e títulos para a concessão do título de especialista em psicologia, especialidade: Neuropsicologia. A prova é composta por 40 questões objetivas, uma prova discursiva (contendo quatro questões) e quatro folhas de rascunho. Para cada uma das questões objetivas, são apresentadas cinco alternativas classificadas com as letras: A, B, C, D e E, havendo apenas uma que corresponde ao quesito proposto. A duração total das provas objetiva e discursiva é de 4 horas e 30 minutos. Os concursos para obtenção do título têm sido realizados uma vez por ano em edital unificado.

1 Nota do editor: Atualmente consta da página citada no artigo o aviso: Conclusão de cursos de especialização credenciados pelo MEC: Seguindo a Ação Civil Pública nº 5994-36.2013.4.01.3800, em trâmite na $20^{\mathrm{a}}$ Vara Federal da Seção Judiciária de Minas Gerais, o Conselho Federal de Psicologia suspendeu o credenciamento e recredenciamento de cursos para concessão do Título Profissional de Especialista em Psicologia, bem como orientou os Conselhos Regionais a emitirem o título aos profissionais que tenham concluído cursos de especialização credenciados pelo MEC, desde que atendam aos demais requisitos previstos na Resolução CFP n ${ }^{\circ}$ 013/2007. Logo, o título de especialista será emitido para psicólogos que concluíram os cursos de pós graduação reconhecidos pelo MEC. 


\section{DESAFIOS SOCIOCULTURAIS ETECNOLÓGICOS: NOVOS TEMPOS NA CIÊNCIA DO CÉREBRO}

O processo de investigação neuropsicológica compreende uma análise quanti-qualitativa das funções cognitivas, funcionais e comportamentais baseando-se em princípios psicométricos rigorosos e bem estabelecidos, tais quais: normatização (validade e aplicabilidade), sensibilidade e especificidade, formas/versões paralelas, custo-benefício (viabilidade) e padronização/adaptação de um instrumento (Witsken et al., 2008). Nos últimos anos, tem-se tido a perspectiva ampliada da avaliação neuropsicológica como uma verificação sociodemógrafica do perfil neuropsicológico, com a identificação de particularidades que atendem a preceitos sociais e culturais de um indivíduo em vista de suas conjunções ambientais (Wajman, 2018). Abordagens integracionistas em neuropsicologia têm considerado não somente a construção de parâmetros psicológicos com base na psicologia "ocidentalizada", mas também está disposta a fundamentar categorias teóricas em termos de culturas específicas das quais podem derivar as capacidades cognitivas (Much, 1995).

De acordo com bases da Neuropsicologia sob a ótica transcultural, o padrão cognitivo pode ser direta e/ou indiretamente influenciado por variáveis relativas aos usos e costumes dos grupos sociais, como as condições do habitat, o modo empregado para gerir a subsistência, os anos de educação e o status social e econômico (Vygotsky et al., 1994). Em um estudo brasileiro, Andrade e Bueno (2007) propuseram investigar a cognição de indígenas do grupo guarani em comparação com seus pares urbanos e discutir a influência de variáveis relativas à cultura sobre o modo de execução dos testes aplicados. Apesar de os resultados quantitativos não demonstrarem diferenças significativas entre os grupos, houve uma tendência estatística do grupo indígena a mostrar maior domínio das tarefas visuais e motoras, e o grupo urbano, das tarefas verbais. Pôde-se concluir, à ocasião, que o grupo indígena pudesse utilizar a cognição de forma mais concreta e intuitiva, em função do estilo peculiar de vida e da maneira ecológica em que desenvolveu suas habilidades (Andrade \& Bueno, 2007).

A exemplo da diversidade histórico-cultural observada no Brasil, a Neuropsicologia na Austrália apresenta adicional desafio em relação às influências de varáveis sociais em detrimento de sua heterogeneidade (Ponsford, 2016). Com uma população que inclui aborígenes e pescadores de origem indígena, a Austrália possui pouco mais de 145 dialetos oficiais diferentes. Nos últimos anos, pesquisadores têm se esforçado em desenvolver e adaptar instrumentos neuropsicológicos para essas populações, tendo-se em vista as diferenças observadas em estudos comparativos (Dingwall \& Cairney, 2011; Dingwall et al., 2012). Como resultado, atualmente a Austrália possui protocolos específicos que reduzem ao máximo os efeitos da escolaridade formal, objetivando a detecção de possíveis mudanças cognitivas através de instrumentos adequados às diferenças populacionais (algo que seria de suma relevância em uma população heterogênea como a brasileira).

De outro lado, à medida que avança o conhecimento nas áreas da saúde, evoluem também as técnicas de avaliação e investigação neuropsicológica. Contraditoriamente, embora diversos campos das neurociências contribuam com significantes modernizações ao longo dos anos, a testagem neuropsicológica segue primariamente calcada na utilização de medidas de desempenho através da chamada avaliação por meio de "lápis e papel". Ciências computacionais e tecnologias da informação experimentaram um salto conceitual com o advento e desenvolvimento de algoritmos e aplicativos para smartphones e tablets, ainda assim, neuropsicólogos rechaçam o uso de computadores em suas avaliações e/ou treinamentos/estimulações motivados, possivelmente, pela carência desses recursos em seus anos de graduação e também pela ausência de produtos comercialmente disponíveis para prática em seus meios (Rabin et al., 2016).

Em paralelo à histórica resistência da psicologia em integrar a tecnologia em sua prática cotidiana (Rabin et al., 2014), surge a adversidade do tecnicismo biomédico e as expectativas geradas por uma sociedade high-tech. Enquanto a psicologia (principalmente clínica e experimental) apoia seus métodos sobre paradigmas da relação cérebrocomportamento em técnicas próprias do século XIX, a Neuropsicologia atualiza sua versão de 1.0 para 2.0, na qual se observa uma releitura de suas bases (Bilder, 2011), seja na renovação de seus dispositivos (leia-se materiais de trabalho) ou na absorção do conhecimento de áreas afins. Inovações tecnológicas expressas por diferentes técnicas de neuroimagem (estrutural, funcional e molecular), aliadas à descrição genética de biomarcadores e o desenvolvimento de novos modelos estatísticos, têm contribuído para o diagnóstico neuropsicológico dos distúrbios cognitivos e comportamentais, e aproximado saberes entre o psicólogo e seus colegas de outras formações.

Naquilo que concerne à relação entre a Neuropsicologia e os avanços tecnológicos provenientes das neurociências, de um modo geral, entram em cena outros aspectos que não os cognitivos, funcionais e comportamentais. Atualmente sabe-se que o sequenciamento de nosso genoma representa um passo essencial no entendimento da biologia humana e no planejamento racional de pesquisas com seres humanos (Kremen et al., 2016). A convergência de dados bioquímicos, de imagem, neuroanatomia, genéticos e clínicos permite prever que estamos muito próximos de uma melhor compreensão das bases biológicas das afecções neuropsiquiátricas que cursam com alterações neuropsicológicas.

Da mesma maneira, uma vez reconhecidas por seus desfechos favoráveis, modalidades terapêuticas adjuvantes acarretam atualizações educacionais por parte 
do neuropsicólogo, em relação à sua compreensão sobre fundamentos psicofarmacológicos. Segundo a literatura médica sobre frentes terapêuticas na clínica psiquiátrica, uma vez combinadas, diferentes estratégias tendem a propiciar melhor resposta terapêutica por parte da pessoa em acompanhamento (Milad \& Quirk, 2012).

Caracteriza outro desafio conceitual que surge tanto na pesquisa neuropsicológica quanto na prática clínica diária, a chamada normatização de escores. Respeitadas as diferenças entre sujeitos (principalmente em populações heterogêneas), pode-se inferir que, em determinadas tarefas (testes, escalas ou baterias), tais sujeitos terão um desempenho diferente da média não devido à subsequente alteração e/ou reserva cognitiva, mas sim em vista de suas capacidades/habilidades de base. Por definição, estes escores fogem à média (suscitando os famosos efeitos fora da curva: solo e teto). Em circunstâncias desta natureza de achado, a adequação do processo neuropsicológico deve respeitar aspectos ecológicos de cada indivíduo (estudo de caso), sem "abrir mão" de pesquisas controladas com grupos, incluindo essas populações particulares. Tais medidas tendem a favorecer a interpretação dos dados e o desenlace clínico, além de tratar com mais veracidade os indicadores de desempenho neuropsicológico (Wajman et al., 2014).

Dessa maneira, a criatividade apoiada em critérios sistematizados poderia superar a estrita prática de traduzir e adaptar ferramentas que foram desenvolvidas, originalmente, em populações estrangeiras (com raízes culturais diferentes), promovendo o desenvolvimento local de medidas cognitivas ajustadas às suas nuances locais. Nessa rota, um exemplo simples, porém marcante, seria a substituição do conceito de escolaridade (ponderada em anos formais dentro da sala de aula), por demanda intelectual mensurada por meio de indicadores não somente cognitivos, mas também socioculturais, pessoais e familiares. Tal noção tem revelado achados interessantes na clínica psicogeriátrica à medida que estabelece parâmetros de funcionamento pré-mórbido mais confiáveis (Apolinario et al., 2012). A principal vantagem, neste caso, seria o tratamento do indivíduo com vistas em seu processo de desenvolvimento/adoecimento de maneira prospectiva e longitudinal, e não somente através de uma impressão pontual de características que atendem ou não a determinados critérios nosológicos, presentes em um manual diagnóstico.

\section{O PERFIL PROFISSIONAL DO NEUROPSICÓLOGO:ALINHAMENTO COM PANORAMA MUNDIAL E A BUSCA POR UMA TAXONOMIA NA FORMAÇÃO E TREINAMENTO EM NEUROPSICOLOGIA NO BRASIL}

O status em que se encontra a prática neuropsicológica e o campo de atuação em Neuropsicologia tem motivado diversos estudos nacionais sobre a área de concentração e o perfil deste profissional, seja em países ocidentais ou orientais (Chan et al., 2016; Hokkanen et al., 2016; Janzen \& Guger, 2016; Kim \& Chey, 2016; Ostrosky \& Velez, 2016; Sakamoto, 2016; Vakil \& Hoofien, 2016). De acordo com um amplo levantamento realizado nas cidades de Bilbao e Granada, ao norte e sul da Espanha, respectivamente, dentre 339 neuropsicólogos que voluntariamente responderam a um questionário. Suas idades variavam entre 22 e 73 anos $(\mathrm{M}=35,8)$, a experiência média ponderada em tempo de atuação era de 7,9 anos, a maioria atuava em hospitaisescola e 77\% eram mulheres (Olabarrieta-Landa et al., 2016). Ainda sobre essa pesquisa, $82 \%$ possuíam mestrado (na área), enquanto $32 \%$ tinham doutorado e $24 \%$ haviam realizado ambos, mestrado e doutorado. Apenas 7,7\% possuíam pós-doutorado. Quase metade (47\%) da amostra referiu fazer parte de alguma associação/sociedade local de Neuropsicologia e aproximadamente $20 \%$ estavam vinculadas a sociedades internacionais (estadunidense, latina e/ou outra europeia).

Em outra pesquisa, desta vez realizada na África do Sul (Truter et al., 2017), 138 pessoas responderam a uma enquete em que a maioria dos achados sociodemográficos se assemelhava ao estudo espanhol, salvo o ambiente de atuação desses neuropsicólogos (56\% atuavam em consultório particular). O questionário foi composto de 67 questões cobrindo sete áreas de interesse: (1) formação profissional, (2) situação atual de trabalho, (3) avaliação e diagnóstico, (4) reabilitação, (5) ensino, (6) pesquisa e (7) aspectos éticos no ambiente de trabalho. Alguns resultados peculiares chamaram a atenção dos pesquisadores, a saber: $20 \%$ nunca haviam recebido supervisão profissional como neuropsicólogos, pouco mais de $80 \%$ trabalhava aproximadamente 25 horas/semana, a média salarial mensal girava em torno de US $\$ 1439,35$ e $79 \%$ subjetivamente atribuíram uma nota seis de satisfação profissional, variando de um a dez sendo que, quanto maior a nota, maior a satisfação. Não obstante, $55 \%$ valiam-se de protocolos neuropsicológicos flexíveis (nos quais prevalece o uso de testes e subtestes sobre baterias fechadas), $66 \%$ utilizavam notas de corte estrangeiras (em geral estadunidense), $40 \%$ compartilhavam seus instrumentos com colegas de profissão (o que incluía realizar fotocópias) e 55\% deles atuavam primariamente na avaliação e diagnóstico diferencial do Transtorno de Déficit de Atenção e Hiperatividade (TDAH).

As áreas de atuação do profissional e as principais dificuldades reportadas pelos neuropsicólogos em sua formação e prática diária foram objeto de estudo na Argentina (Fernandez et al., 2016), em uma ampla pesquisa internacional envolvendo 39 países, nos cinco continentes (ArangoLasprilla et al., 2017). Em nosso país vizinho, produto das entrevistas dos 135 respondentes incluídos e identificados 
como neuropsicólogos, seis grupos de pacientes foram elencados representando as áreas nas quais esses profissionais atuavam mais frequentemente: (1) derrames/neurovascular (36\%), (2) demência/neurodegenerativa (30\%), (3) TDAH (23\%), depressão/transtornos do humor (21\%), convulsões/ neuroepilepsia (21\%) e trauma cranioencefálico $(20 \%)$. Quando indagados sobre as principais barreiras encontradas em sua prática profissional, os indivíduos inquiridos relataram: A) ausência/carência de dados normatizados para seu país (64\%), B) alto valor para obtenção dos instrumentos disponíveis $(60 \%)$, C) falta de estudos para validação transcultural de testes em seu país (50\%) e D) inadequação diante da realidade populacional local (29\%). Para aproximadamente $10 \%$ dos entrevistados, os instrumentos eram muito longos e/ou difíceis de administrar/aplicar.

Paralelamente aos obstáculos citados, cabe ao profissional em Neuropsicologia o conhecimento e domínio de alguns assuntos que orbitam a área das neurociências. No Brasil, assim como em outros países sul-americanos, tais requisições não são completamente estabelecidas, seja nos aspectos da avaliação, diagnóstico, reabilitação, ensino ou pesquisa (Arango-Lasprilla, et al. 2017). Conforme cronograma de alguns processos de acreditação e cursos de formação em Neuropsicologia deveriam contemplar o rol de disciplinas/ matérias exigidas para prova de título ou pós-graduação/ especialização em Neuropsicologia, temas de acordo com sua categoria central e suas especificações periféricas (Tabela 1).

Tabela 1

Conhecimento Básico Exigido para Obtenção do Título em Neuropsicologia

\begin{tabular}{|c|c|}
\hline Categoria & Especificações \\
\hline $\begin{array}{l}\text { Processos neuropsicológicos } \\
\text { básicos }\end{array}$ & Domínios cognitivos \\
\hline Substratos neuroanatômicos & $\begin{array}{l}\text { Correlação cérebro- } \\
\text { comportamento }\end{array}$ \\
\hline Modelos neuropsicológicos & Sistemas e subsistemas \\
\hline Alterações neuropsicológicas & $\begin{array}{l}\text { Estáveis, sequelares e } \\
\text { degenerativas }\end{array}$ \\
\hline Psicometria & Fundamentos estatísticos \\
\hline Pesquisa e ética profissional & Metodologia, proteção e de dados \\
\hline Semiologia clínica & Síndrome, topografia e etiologia \\
\hline Elaboração de laudos/relatórios & Reporte \\
\hline Intervenções & $\begin{array}{l}\text { Programação de } \\
\text { acompanhamento }\end{array}$ \\
\hline
\end{tabular}

Diversos países vêm desenvolvendo, cada qual à sua maneira, um modelo padronizado para o ensino e treinamento em Neuropsicologia. Na vanguarda, a América do Norte representada pelas seguintes associações/ sociedades: American Board of Clinical Neuropsychology (ABCN), Association of Postdoctoral Programs in Clinical Neuropsychology, International Neuropsychological Society, National Academy of Neuropsychology, Society for Clinical Neuropsychology e Sports Neuropsychology
Society possui uma organizada rede de recursos para recolhimento de informações e diretrizes para formação de novos profissionais com atuação em Neuropsicologia. Em seus diretórios é possível encontrar, além de credenciais para admissão em programas de certificação, uma distribuição entre estados e províncias acerca de salários médios para cada tipo de profissional (Learn how to Become, n.d.). Atualmente, de acordo com a $\mathrm{ABCN}$, existem aproximadamente 150 cursos reconhecidos e recomendados para formação continuada em Neuropsicologia nos Estados Unidos (Psychology Career Center, 2014).

Em nosso meio, apesar de sua limitada representatividade diante da variedade dos campos de atuação, das diferenças regionais em relação a seu poder aquisitivo e da quantidade de psicólogos atuantes na área da Neuropsicologia, é possível encontrar por meio do Site Nacional de Empregos (SINE, 2017) a média salarial na função de neuropsicólogo em instituições privadas de saúde e educação. Em banco de dados com 182 salários cadastrados nos últimos 12 meses e na relação entre porte da empresa (Pequena: até 99 funcionários; Média: entre 100-499 e Grande: +499) versus experiência profissional (Trainee: até dois anos de experiência; Júnior: 2-4; Pleno: 4-6; Sênior: 6-8 e Master: +8 ), constam os seguintes indicadores a respeito de honorários: (Pequena): R \$ 1774,74, R\$ 2218,43, R\$ 2773,04, R \$ 3466,3, R\$ 4332,88, (Média): R \$2307,17, R\$ 2883,96, R\$ 3604,95, R\$ 4506,19, R\$ 5632,74 e (Grande): $\mathrm{R} \$ 2999,32, \mathrm{R} \$ 3749,15, \mathrm{R} \$ 4686,44, \$ 5858,05, \mathrm{R} \$ 7322,56$. Vale ressaltar que as informações contidas no diretório SINE dizem respeito a uma prestação de utilidade pública tendo em consideração o mercado brasileiro de trabalho e não possui relação com instituições governamentais e/ou quaisquer outros órgãos públicos.

Historicamente, com exceção de poucos centros de referência e destaque (em geral, norte-americanos ou europeus), a Neuropsicologia Clínica falha no encargo de clara e consistentemente definir princípios convencionados e bem estabelecidos para educação e treinamento na área. Em oposição, o esforço e a colaboração de profissionais e organizações em caracterizar sistematicamente as diretrizes para formação e educação em Neuropsicologia têm lançado luz a um modelo taxonômico em prol de uma consonância para busca de parâmetros disciplinares. A partir de documentos tomados em conjunto e analisados por meio de juntas especializadas, consagrou-se a primeira iniciativa para criação de um modelo de educação e treinamento em Neuropsicologia clínica (Sperling et al., 2017). Tal determinação estabeleceu definições hierárquicas de ensino e treinamento nos campos acadêmico, clínico e de pesquisa, nos quatro seguintes estágios profissionalizantes: (1) estágio (com ao menos $50 \%$ do conteúdo relacionado à Neuropsicologia e $30 \%$ de atividade clínica supervisionada), (2) pós-graduação (ao menos dois cursos dedicados à Neuropsicologia, além de dissertação, tese e publicações), (3) pós-doutoramento (dois anos com dedicação exclusiva em 
prática clínica e colaboração em projetos multidisciplinares) e (4) licenciatura plena (engajamento na disseminação e educação continuada em Neuropsicologia).

Por fim, reguladores da formação e atuação em Neuropsicologia, cabe às autarquias federais e aos conselhos regionais de classe profissional (e também a acadêmicos, profissionais e associações/sociedades interessados no avanço e crescimento da Neuropsicologia brasileira) a constante busca pela parametrização da formação em Neuropsicologia e qualidade dos currículos oferecidos pelas instituições de ensino, além do investimento em forças-tarefa para o desenvolvimento e normatização de instrumentos neuropsicológicos, por meio de comitês e agências regulamentadoras.
Desse modo, em 2010, propôs-se no Conselho Regional de Psicologia 8 que neuropsicólogos fossem devidamente informados e estimulados para que passassem a fazer uso de ferramentas normatizadas e disponíveis na literatura científica nacional, dada a consolidação destes instrumentos em áreas mais abrangentes que igualmente fazem interface com a neurociência. Desta maneira, aspectos multiprofissionais se incluem no método de avaliação em Neuropsicologia, contemplando congregar e cooperar com diferentes profissionais e pesquisadores nas áreas biológicas e da saúde, promovendo a titulação padronizada dos profissionais psicólogos em todas as subdivisões regionais, mas também fomentando e promovendo a criação de grupos de trabalho.

\section{CONSIDERAÇÕES FINAIS}

A Neuropsicologia é, em sua essência, uma ciência aplicada e, como diversas outras disciplinas, a Neuropsicologia vem testemunhando os efeitos de uma vertiginosa evolução tecnológica, provocando a necessidade de atualizações e reformulações em relação às suas raízes conceituais, oferecendo fundamentação aos acadêmicos, suporte aos profissionais e primando pelo bem-estar das pessoas que dela se beneficiam. Igualmente, a Neuropsicologia se depara com os desafios que acompanham as inexoráveis transformações socioculturais observadas em nossa sociedade, incluindo, dentre outras, a necessidade de se ajustar ao perfil cognitivo e comportamental de nossa heterogênea população, o que inclui "novos" estratos como a infância encurtada, a adolescência esticada e a longevidade superada.

Paradoxalmente, quanto mais a Neuropsicologia foi, naturalmente, se (re)aproximando das ciências biológicas, mais perceptível tem sido o efeito da pressão ambiental a qual está exposta. Fundamentos básicos do conhecimento acerca do organismo fizeram com que o psicólogo especialista em Neuropsicologia ampliasse seu entendimento adaptando-se às novas contingências sociais e científicas, em busca de uma psicologia baseada em evidências. Desta maneira, constitui o "Know-How" deste especialista, fundamentos básicos e/ou avançados sobre temas complementares, tais quais: introdução à Neuropsicologia (histórico), Neuroanatomia, Neurofisiologia, Neuropatologia (síndromes neurológicas), Psicofarmacologia, Neuroimagem (técnicas subsidiárias), sistemas cognitivos, desenvolvimento neuropsicológico, semiologia em Neuropsiquiatria clínica, Metodologia Científica, modelos estatísticos, contextos da Neuropsicologia (áreas de atuação), Psicometria (avaliação neuropsicológica), laudos/relatórios neuropsicológicos e técnicas de reabilitação.

Pesquisas recém-realizadas e previamente citadas vêm demonstrando tendência e premência em caracterizar a atividade do neuropsicólogo nos mais diversos locais nos quais a mesma é ensinada, praticada e difundida. Igualmente, no Brasil, faz-se necessário uma taxonomia acerca do ofício do profissional especialista em Neuropsicologia, seja em relação à sua formação ou de sua atuação profissional. Tal exercício curricular atenderia não somente a uma necessidade particular da Neuropsicologia, mas também a agenda da profissão do psicólogo uma vez que outras subáreas poderiam se favorecer da matéria aqui interrogada, visando o progresso da psicologia brasileira como um todo.

Como entendida por Cícero, podemos dizer que, em grande parte, a história é a matéria "mestra da vida" (Catroga, 2006). Registrar e transferir acontecimentos marcantes oferece suporte intelectual para que as pessoas, ao longo de suas gerações, sejam capazes de aprender com experiências passadas e superar desafios atuais através da contínua reciclagem de saberes. A história da Neuropsicologia deve, então, fazer jus ao seu papel presente de orientar para um tempo futuro, não se limitando a apresentar uma soma de eventos isolados, mas acima de tudo servindo como um parâmetro retroativo para um movimento da própria dinâmica científica e social em que se insere na atualidade. Suposições outrora formidáveis foram superadas por novas teorias e foi justamente a descrição dos métodos utilizados à ocasião que permitiu aos experimentalistas contemporâneos uma tese original, que superasse os desafios da época e viesse a contribuir com uma nova perspectiva.

O presente artigo teve como objetivo central apresentar o desenvolvimento histórico da Neuropsicologia, em especial no Brasil, realçando fundamentos teórico-metodológicos essenciais, sua conjuntura regulamentária vigente e perspectivas futuras na criação de diretrizes para formação profissional. Apesar de este estudo limitar-se a uma discussão teórica acerca dos temas abordados, estudantes e profissionais podem se beneficiar do conteúdo apresentado, em busca do desenvolvimento e execução de estudos controlados que atendam às questões da Neuropsicologia como especialidade e promovam seu avanço através da disseminação e compreensão entre colegas de profissão e também de áreas correlatas. 


\section{REFERÊNCIAS}

Andrade, V. M., \& Bueno, O. F. A. (2007). Neuropsicologia transcultural: Grupo indígena guarani. Estudos de Psicologia, 12(3), 253-258. https://doi.org/10.1590/S1413294X2007000300007

Arango-Lasprilla, J. C., Stevens, L., Morlett, P. A., Ardila, A., \& Rivera, D. (2017). Profession of neuropsychology in Latin America. Applied Neuropsychology: Adult, 24(4), 318-330. https://doi.org/10.1080/23279095.2016.1185423

Apolinario, D., Braga, R. C. O. P., Magaldi, R. M., Busse, A. L., Campora, F., Brucki, S., \& Lee, S. D. (2012). Short Assessment of Health Literacy for Portuguese-speaking Adults. Revista de Saúde Pública, 46(4), 702-11. https://doi.org/10.1590/S003489102012005000047

Bilder, R. M. (2011). Neuropsychology 3.0: Evidencebased science and practice. Journal of the International Neuropsychological Society, 17, 7-13. https://doi.org/10. 1017/S1355617710001396

Boring, E. G. (1950). A history of experimental psychology $\left(2^{\text {nd }}\right.$ ed.). Prentice-Hall.

Bruce, C. (1985). On the origin of the term Neuropsychology. Neuropsychologia, 23, 813-814. https://doi.org/10.1016/00283932(85)90088-0

Carrilho P. E. M. (2009). Síndromes de desconexão: Um breve apanhado histórico e o importante legado de Norman Geschwind. Revista Varia Scientia, 7(14), 55-66.

Catroga, F. (2006). Ainda será a história mestra da vida? Estudos Ibero-Americanos 2, 7-34. https://doi.org/10.15448/1980864X.2006.s. 1347

Chan, A. S., Sze, S. L., Cheung, M. C., \& Han, Y. M. (2016). Development and application of neuropsychology in Hong Kong: Implications of its value and future advancement. Clinical Neuropsychology, 30(8), 1236-1251. https://doi.org /10.1080/13854046.2016.1196732

Centro Latino-Americano e do Caribe de Informação em Ciências da Saúde. (n.d.). BVS-Psicologia Brasil. Terminologias. http://newpsi.bvs-psi. org.br/cgi-bin/wxis 1660.exe/iah/?IsisScript=iah/iah. $\mathrm{xis} \&$ lang $=\mathrm{P} \&$ base $=$ TERMINOLOGIA

Conselho Federal de Psicologia. (2007). Título de especialista. https://site.cfp.org.br/servicos/titulo-de-especialista/

Conselho Federal de Psicologia. (2018). Seja bem-vindo ao Sistema de Avaliação de Testes Psicológicos (SATEPSI). http://satepsi. cfp.org.br/.

Conselho Regional de Psicologia 8. (2010). A atuação do Psicólogo especialista em Neuropsicologia dentro do contexto científico brasileiro - Uma reflexão sobre Exame Neuropsicológico com referência às Resoluções do CFP 25/2001 (Regulamenta Testes Psicológicos), 002/2003 (que revoga a anterior) e 02/2004 (Institui a Especialidade em Neuropsicologia). https://crppr. org.br/wp-content/uploads/2019/10/277.pdf

Dias, M. J. M. (2013). Homenagem à Beatriz Helena Whitaker Léfévre. IBNEC.

Dingwall, K. M., \& Cairney, S. (2011). Detecting psychological symptoms related to substance use among Indigenous Australians. Drug and Alcohol Review, 30, 33-39. https://doi. org/10.1111/j.1465-3362.2010.00194.x

Dingwall, K. M., Maruff, P., Clough, A. R., \& Maruff, M. (2012). Factors associated with continued solvent use in Indigenous petrol sniffers following treatment. Drug and Alcohol Review, 31, 40-46. https://doi.org/10.1111/j.1465-3362.2010.00279.x

Fernandez, A. L., Ferreres, A., Morlett-Paredes, A., Rivera, D., \& Arango-Lasprilla, J. C. (2016). Past, present, and future of neuropsychology in Argentina. Clinical Neuropsychology, 30(8), 1154-1178. https://doi.org/10.1080/13854046.2016.1 197313
Gounden, Y., Hainselin, M., Cerrotti, F., \& Quaglino, V. (2017). Dynamic and functional approach to human memory in the brain: A clinical neuropsychological perspective. Frontier Psychology, 4(8), 688. https://doi.org/10.3389/ fpsyg.2017.00688

Hokkanen, L., Nybo, T., \& Poutiainen, E. (2016). Neuropsychology in Finland - Over 30 years of systematically trained clinical practice. Clinical Neuropsychology, 30(8), 1214-1235. https:// doi.org/10.1080/13854046.2016.1196733

IBOPE Opinião. (2004). Pesquisa de Opinião IBOPE - Psicólogo Brasileiro. https://site.cfp.org.br/publicacao/pesquisa-deopinio-ibope-psiclogo-brasileiro/

Instituto Brasileiro de Neuropsicologia e Comportamento. (2018). Sobre o IBNEC. http://www.ibnec.com.br/site/sobre.php

Janzen, L. A., \& Guger, S. (2016). Clinical neuropsychology practice and training in Canada. Clinical Neuropsychology, 30(8), 1193-1206. https://doi.org/10.1080/13854046.2016.1 175668

Kapczinski, N. S., \& Narvaez, J. C. M. (2008). Neuropsicologia, teoria e prática. Revista Brasileira de Psiquiatria, 30(3) 304. https://doi.org/10.1590/S1516-44462008000300028

Kim, M. S., \& Chey, J. (2016). Clinical neuropsychology in South Korea. Clinical Neuropsychology, 30(8), 1325-1334. https:// doi.org/10.1080/13854046.2016.1212097

Kremen, W. S., Panizzon, M. S., \& Cannon, T. D. (2016). Genetics and neuropsychology: A merger whose time has come. Neuropsychology, 30(1), 1-5. https://doi.org/10.1037/ neu0000254

Lefèvre, B. H. (1985). Antonio F. Branco Lefèvre: Teses, artigos inéditos e crônicas. Sarvier.

Lezak, M. (1995). Neuropsychological assessment. Oxford University Press.

Luria, A. R. (2005). Autobiography of Alexander Luria: A dialogue with the Making of Mind. Lawrence Erlbaum Associates.

Mäder, J. M. (1996). Avaliação neuropsicológica: Aspectos históricos e situação atual. Psicologia: Ciencia \& Profissão, 16(3), 12-18. https://doi.org/10.1590/S141498931996000300003

Milad, M. R., \& Quirk, G. J. (2012). Fear extinction as a model for Translational Neuroscience: Ten Years of Progress. Annual Review of Psychology, 63, 129-151. https://doi.org/10.1146/ annurev.psych.121208.131631

Miotto, E. C. (2007). Neuropsicologia: Conceitos fundamentais. In E. C. Miotto, M. C. Lucia, \& M. Scaff (Eds.), Neuropsicologia e as interfaces com as neurociências (pp. 137-142). Casa do Psicólogo.

Much, N. (1995). Cultural psychology. In J.A. Smith, R. Harré, \& L. V. Langenhove (Eds.), Rethinking psychology (pp. 97121). Sage.

Mueller, F. L. (1968). História da psicologia (D. Penna, Trad.). Companhia Editora Nacional.

Learn how to Become. (n.d.). Neuropsychology Degrees \& Careers: How to Become a Neuropsychologist. http://www. learnhowtobecome.org/psychologist/neuropsychologist/

Olabarrieta-Landa, L., Caracuel, A., Pérez-García, M., Panyavin, I., Morlett, P. A., \& Arango-Lasprilla, J. C. (2016). The profession of neuropsychology in Spain: Results of a national survey. Clinical Neuropsychology, 30(8), 1335-1355. https://doi.org/ 10.1080/13854046.2016.1183049

Ostrosky, S. F., \& Velez, G. A. (2016). Neuropsychology in Mexico. Clinical Neuropsychology, 30(8), 1296-1304. https://doi.org/ $10.1080 / 13854046.2016 .1226387$

Ponsford, J. (2016). The practice of clinical neuropsychology in Australia. Clinical Neuropsychology, 30(8), 1179-1192. https:// doi.org/10.1080/13854046.2016.1195015 
Psychology Career Center. (2014). Accreditation in Psychology. Retirado de https://www.psychologycareercenter.org/ accreditation.html

Rabin, L. A., Spadaccini, A. T., Brodale, D. L., Grant, K. S., Elbulok-Charcape, M. M., \& Barr, W. B. (2014). Utilization rates of computerized tests and test batteries among clinical neuropsychologists in the United States and Canada. Professional Psychology: Research and Practice, 45, 368. https://doi.org/10.1037/a0037987

Rabin, L. A., Paolillo, E., \& Barr, W. B. (2016). Stability in test-usage practices of clinical neuropsychologists in the United States and Canada over a 10-year period: A followup survey of INS and NAN members. Archives of Clinical Neuropsychology, 31(3), 206-230. https://doi.org/10.1093/ arclin/acw007

Rourke, B. P., \& Murji, S. (2000). A history of the International Neuropsychological Society: The early years. Journal of the International Neuropsychological Society, 6, 491-509.

Sakamoto, M. (2016). Neuropsychology in Japan: History, current challenges, and future prospects. Clinical Neuropsychology, 30(8), 1278-1295. https://doi.org/10.1080/13854046.2016.1 204012

Site Nacional de Empregos. (2017). Média salarial para neuropsicólogo. https://www.sine.com.br/media-salarial-paraneuropsicologo

Sperling, S. A., Cimino, C. R., Stricker, N. H., Heffelfinger, A. K., Gess, J. L., Osborn, K. E.., \& Roper, B. L. (2017). Taxonomy for education and training in clinical neuropsychology: Past, present, and future. Clinical Neuropsychology, 10, 1-12. https://doi.org/10.1080/138 54046.2017.1314017
Spreen, O., \& Strauss, E. (1991). A compendium of neuropsychological tests: Administration, norms, and commentary. Oxford University Press.

Truter, S., Mazabow, M., Morlett, P. A., Rivera, D., \& ArangoLasprilla, J. C. (2017). Neuropsychology in South Africa. Applied Neuropsychology: Adult, 21, 1-12. https://doi.org/10 $.1080 / 23279095.2017 .1301453$

Vakil, E., \& Hoofien, D. (2016). Clinical neuropsychology in Israel: History, training, practice and future challenges. Clinical Neuropsychology, 30(8), 1267-1277. https://doi.org/10.1080 /13854046.2016.1175667

Vygotsky, L. S. (1991). A formação social da mente (4ª ed.). Livraria Martins Fontes.

Vygotsky, L. S., Luria, A. R., \& Leontiev, N. A. (1994). Linguagem, desenvolvimento e aprendizagem. Ícone.

Wajman, J. R. (2018). Evolutionary traits of human cognition: An introductory essay on the interface between Cultural Neuroscience and Neuroanthropology. International Journal of Brain and Cognitive Sciences, 7(1), 17-29. https://doi. org/10.5923/j.ijbcs.20180701.03

Wajman, J. R., Oliveira, F. F., Marin, S. M. C., Schultz, R. R., \& Bertolucci, P. H. F. (2014). Is there correlation between cognition and functionality in severe dementia? The value of a performance-based ecological assessment for Alzheimer's disease. Arquivos de Neuropsiquiatria, 72(11), 845-850. https:// doi.org/10.1590/0004-282X20140145

Witsken, D., D'Amato, R. C., \& Hartlage, L. C. (2008). Understanding the past, present, and future of clinical neuropsychology. In R. C. D'Amato \& L. C. Hartlage (Eds.), Essentials of neuropsychological assessment: Rehabilitation planning for intervention (pp. 1-30). Springer. 